\title{
Correspondence
}

\section{COMPULSORY NOTIFICATION OF PRION DISEASES IN BRAZIL: WHAT HAS CHANGED SINCE 2005?}

\section{To the Editor}

Recently, in an article published by Dementia \& Neuropsychology, Martins et al. (2007) ${ }^{1}$ screened mutations and polymorphisms at the prion gene (PRNP) in 35 cases of Creutzfeldt-Jakob disease (CJD) from 13 Brazilian states, reported to the Health Surveillance Secretariat/Ministry of Health (SVS/MS), between 2005 and 2007. This study is highly important because prion diseases $(P D)$ are linked to mutations in the PRNP while some polymorphisms at the same gene are considered risk factors for these groups of disorders. Numbering among them is the polymorphism of the codon 129 which has been described in other articles as the most significant risk factor for such disorders., ${ }^{2,3}$

After thoughtful reading we would like to discuss some points that we feel should be addressed. One of these is a minor misunderstanding in the Abstract and Results sections of the main text. Regarding the first, the authors mentioned that "twenty seven cases (74\%) were classified as possible sCJD while $51 \%$ were classified as probable sCJD". However, these $74 \%$ (which corresponds to 26 and not 27 cases as stated in the Article) represent the total of SCJD (possible + probable). In Results, the statement "sCJD possible (probable + possible)" is inadequate, because it suggests that cases of "possible sCJD" is the sum of possible and probable cases. However, these small mistakes do not detract from the importance of this work.

Analyzing the epidemiological data, we observed that only 35 cases reported in two years by SVS/MS included complementary examinations of blood and / or cerebral spinal fluid (CSF). Three of these were studied with Neuropathologic analysis, but only two confirmed the presence of prion protein aggregate. These procedures are crucial to exclude other etiologies and to better analyze these disorders which often presenting with clinical heterogeneity.

The data gathered so far by Martins et al. are highly relevant and promising, showing that $89 \%$ of the compulsory notification forms were filled out properly, enriching epidemiological analysis of this neglected disorder.

Most of the biological samples examined to confirm this diagnosis were sent to reference centers in São Paulo, showing that only one Brazilian state is leading this process.
However, we should have at least one reference center per region, considering the continental dimensions of Brazil. This is especially true if we consider the potential consequences to the Brazilian Health system and Economy in the event of a possible epidemic.

Last December, at the VI RPDA (Reunião de Pesquisadores em Doença de Alzheimer e Desordens Correlacionadas) ${ }^{4}$ we reported that, up until 2004, the epidemiology about CJD was not reliable because there were many communication problems between the state secretariats of health and the Health Ministry. Nevertheless, in 2005, the Health Ministry included PD in the compulsory notification list, which requires health care professionals to notify all suspected cases to public departments of health in order to establish groups of epidemiological surveillance, reinforcing the PD epidemiology. ${ }^{5}$

Between 2006 and 2007, we visited the state secretariats of health in Minas Gerais (MG), Pernambuco (PE), Rio Grande do Norte (RN) and São Paulo (SP) and noted that only São Paulo presented an active and organized group of epidemiological surveillance for $P D$, adopting what is considered standard model.

After the visits, we maintained contact with the RN and PE secretariats, but so far the scenario is the same.

Currently, the Secretariat of Health in Pernambuco is concerned about forming a group of epidemiological surveillance for these kinds of diseases. A lecture was held last February with the presence of various members of the State Health technical staff to increase awareness for technical management of $P D$.

After 3 years of effort to form a network of epidemiological surveillance for $P D$, the public health services still face several challenges including lack of personnel able to identify clinical and laboratorial aspects involved with $P D$, and scant resources to supply the necessary technical procedures to increase diagnosis accuracy, besides the centralization of the study in São Paulo. The sum of all these factors compromise the setting up of $P D$ study centers across the country.

The study by Martins et al., reviewed in this article, reflects the dedication of the Ludwig Institute for Cancer Research in São Paulo, especially by Dr Vilma Martins, during the last ten years studying the prion protein and its related diseases, which are improperly studied in some countries including Brazil, due to the obstacles previously described. 


\section{References}

1. Martins, VR, Gomes HR, Chimelli L, Rosemberg S, Landemberger MC. Prion diseases are under compulsory notification in Brazil. Dement Neuropychol 2007;1:347-355

2. Mead S, Stumpf MP, Whitfield J, et al. Balancing selection at the prion protein gene consistent with prehistoric kurulike epidemics. Science 2003; 300(5619):640-643

3. Erich VP, Addas-Carvalho M, Souza DPC, Olalla TSS, Olenscki GCO. Genotype frequencies at codon 129 of the Prion Protein Gene in Brazil: implications in susceptibility to variant Creutzfeldt-Jakob compared to European and Asian populations. European Journal of Epidemiology 2005;20:593-595.

4. Cunha JEG, Oliveira JRM. How Reliable is the CJD epidemiology in Brazil? Dement Neuropsychol 2007;1(suppl 2):26.

5. Gattás VL, Lima Neto ASL, Dimech GS, et al. New variant of Creutzfeldt-Jakob (vCJD) disease and other human prion diseases under epidemiological surveillance in Brazil. Dement Neuropychol 2007;1:339-346.

José Eriton Gomes da Cunha ${ }^{1}$, João Ricardo M. Oliveira ${ }^{2}$ ${ }^{1}$ Biological Sciences Center -Undergraduate program- Federal University of Pernambuco (UFPE). ${ }^{2}$ Department of Neuropsychiatry and Keizo Asami Laboratory (LIKA) - Federal University of Pernambuco (UFPE), Recife - Pernambuco, Brazil.

\section{Reply from the authors}

Regarding the comments from Gomes da Cunha \& Oliveira we acknowledge the error in the Abstract of our manuscript where a total of twenty six (as described in the results) instead of twenty seven cases were classified as possible sCJD. In their letter the authors also mentioned our statement in the results section that possible CJD may represent the sum of possible and probable sCJD. In fact, in that specific paragraph we were describing Table 1. From the total cases of notified patients $74 \%$ were initially classified, based on clinical parameters, as possible sCJD. When these patients were analyzed for positive levels of 14.3.3, $51 \%$ or the total were confirmed as probable sCJD while $23 \%$ did not fulfill criteria for this classification and contin- ued to be classified as possible sCJD. Indeed, in this specific case (Table 1 ) the group representing $74 \%$ of the patients initially classified as possible sCJD, contains patients that were later classified as probable CJD (51\%) where 23\% of the patients remained classified as possible sCJD.

We agree with Gomes da Cunha \& Oliveira that there is a need for improved communication between some State Secretariats and the Ministry of Heath. However, we have handled notified cases, and had samples submitted for biochemical analysis, from all over the country indicating that a working minimal framework is in place. Nevertheless, there is a special need to make an effective statement on compulsory notification of these diseases to the medical community, particularly to neurologists and psychiatrics. Indeed, proper notification of suspected cases is the first step toward establishing the epidemiological surveillance of prion diseases in Brazil.

On the other hand, we disagree with Gomes da Cunha \& Oliveira that reference centers for diagnosis must be created in each Brazilian region. We know that the incidence of these diseases is about 1 in 1,000,000 inhabitants/year. Given the IBGE (Brazilian Institute of Geography and Statistics) have estimated that the Brazilian population in 2004 was around 180,000,000, we expected to have 180 to 200 cases of CJD a year in Brazil. This is a very low number of patients to justify investment in acquiring equipments and training personnel in different regions. Costs will be much lower if the diagnosis is concentrated at one reference center, independently of where it is located in Brazil. This is exactly the approach adopted in other counties such as Argentina, USA, Canada, England and Germany.

Indeed, we have to concentrate effort in each State to identify, notify and follow all the possible cases of prion disease in Brazil. The adequate epidemiological surveillance of patients with prion diseases shall allow Brazil to contribute to clinical trials developing drugs to treat these devastating diseases.

Vilma Regina Martins, Hélio Rodrigues Gomes, Leila Chimelli, Sergio Rosemberg, Michele Christine Landemberger 